\title{
REPRESENTACIONES DE LOS ESTUDIANTES SOBRE LAS MODALIDADES $Y$ PRÁCTICAS EVALUATIVAS EN LA ASIGNATURA DE HISTORIA, GEOGRAFİA Y CIENCIAS SOCIALES EN ESTABLECIMIENTOS EDUCACIONALES PÚBLICOS, VULNERABLES Y FRONTERIZOS
}

\author{
STUDENT REPRESENTATIONS ON ASSESSMENT MODALITIES AND PRACTICES \\ IN THE SUBJECT OF HISTORY, GEOGRAPHY AND SOCIAL SCIENCES, IN \\ PUBLIC EDUCATION INSTITUTIONS, VULNERABLE AND FRONTIER
}

\author{
Eduardo Alejandro Velásquez Parraguez ${ }^{1}$ \\ Raúl Antonio Bustos González ${ }^{2}$ \\ María del Mar Duran Bellonch ${ }^{3}$
}

Aceptado: $30 / 11 / 2020$

Publicado online: $14 / 12 / 2020$

\begin{abstract}
RESUMEN
La investigación analiza las representaciones de los estudiantes sobre las modalidades y prácticas evaluativas utilizadas en la asignatura de Historia, Geografía y Ciencias Sociales, de establecimientos públicos, vulnerables de una región fronteriza. El estudio es cualitativo, empleando técnicas de corte interpretativo a fin de explorar las representaciones entorno a las prácticas evaluativas. La recolección de información se estableció a través de entrevistas en profundidad. Los resultados informaron que los principales temas identificados fueron una concepción dinámica de aprendizaje, una mirada compleja sobre la evaluación que incluye valores actitudinales y una representación del docente como agente que flexibiliza los criterios institucionales de evalución en atención a los objetivos de aprendizaje.
\end{abstract}

Palabras clave: aprendizaje, conocimiento, estudiante-profesor, evaluación, contexto de frontera..

\begin{abstract}
The research explores the representations of the students on the modalities and evaluative practices used in the subject of History and Geography, of public, vulnerable establishments of a border region. The study is qualitative, employing interpretive techniques in order to
\end{abstract}

\footnotetext{
${ }^{1}$ Magíster en Gestión y Liderazgo Educacional en la Universidad Central, Santiago de Chile. Magíster en Desarrollo Curricular y Proyectos Educativos en la Universidad Andrés Bello, Santiago de Chile. Académico, investigador Coordinador de Prácticas Pedagógicas Pedagogía en Historia y Geografía Universidad de Tarapacá y Jefe de Unidad Técnico Pedagógica en el sistema escolar, Arica-Chile. evelasquez@academicos.uta.cl. (D) 0000-0002-7434-4039

${ }^{2}$ Profesor de Historia y Geografía de la Universidad de Tarapacá, Magíster en desarrollo subregional de la Universidad Arturo Prat y Doctor en Educación y Cultura en América Latina de la Universidad de Artes y Ciencias Sociales y Doctor en Educación de la Universidad Autónoma de Barcelona. Académico del Departamento de Educación y Coordinador de Desarrollo Académico de la Vicerrectoría Académica de la Universidad de Tarapacá. rbustos@academicos.uta.cl (D) 0000-0002-2363-1919

3 Licenciada en Filosofía y Letras y Doctora en Pedagogía por la UAB. Profesora del Departamento de Pedagogía Aplicada, especializada en formación para la constitución y gestión de grupos y equipos de trabajo en contextos educativos. MariadelMar.Duran@uab.cat. (D)0000-0003-1425-3570
} 
explore the representations around the evaluative practices. Information gathering was established through in-depth interviews. The results reported that the main issues identified were a dynamic conception of learning, a complex look at evaluation that includes attitudinal values and a representation of the teacher as an agent that makes institutional evaluation criteria more flexible in response to learning objectives

Key words: learning, knowledge, student-teacher, evaluation, frontier context.

\section{INTRODUCCIÓN}

Desde el último cuarto de siglo pasado asistimos a una serie de cambios sociales y demográficos que han tenido repercusiones en las instituciones educativas, teniendo como principales consecuencias impacto en la estructura, funciones y actores del entorno educativo, lo que ha traído una trasnformación de las actitudes de los agentes sociales que intervinen en el espacio educativo. En este sentido, los juicios y representaciones de docentes y alumnos reproducen cambios de primer orden dentro de la trasnformaciones sociales del espacio educativo, lo que tiene repercusiones en la praxis y el trabajo.

Habiéndose producido dichos cambios no solo a nivel de las condiciones materiales, sino de la metodología y epistemología, podemos afirmar que han surgido nuevas formas de pensar la evaluación en el aula desde la acción social de los agentes. Así, se debe otorgar una importancia fundamental a la díada docente-alumno como capaz de construir una idea de la práctica educativa. Ante esta nueva realidad, es necesaria una reestructuración de la evaluación y de las metodologías de enseñanza, tomando en cuenta las nuevas formas de concebir la relación del alumno con el aprendizaje y cómo aquél se apropia de este.

En el caso de la Región de Arica y Parinacota desde el enfoque educativo, se presentan características atrayentes de señalar, particularmente en relación a los resultados de la Evaluación Docente aplicada a los profesores que se desempeñan en establecimientos públicos. Unos de los módulos que tienen que rendir es el portafolio docente. En dicho módulo, la tarea de "generar una evaluación de calidad" sólo el 25 \% de los docentes de la comuna rural de Putre logró el estándar, a su vez, en la comuna rural de Camarones el logro es de un $16 \%$. En cuanto al ítem "Reflexión a partir de los resultados de la evaluación", el resultado es aún más descendido, exibiéndose una comuna (Camarones) en la que ningún docente logra lo esperado (Marín, 2016). Lo señalado apunta a la manera en que los docentes de la región enfrentan el desafío de evaluar la marcha del proceso educativo de sus estudiantes.

De acuerdo a lo anterior, se plantea a través del presente trabajo analizar desde una mirada cualitativa las representaciones y juicios que tienen los estudianes en torno a las modalidades y prácticas evaluativas empleadas en la asignatura de HistoriaGeografía y Ciencias Sociales.

El estudio es parte de un proyecto de investigación Fondecyt: INI 11180044, en base a la aplicación de entrevistas en profundidad a estudiantes de los establecimientos seleccionados con el fin de reconocer en los participantes, modelos identificables en la representación del proceso evaluativo. La reducción de los datos se realiza atendiendo a criterios temáticos.

\section{METODOLOGÍA}

El presente artículo pretende analizar desde la perspectiva cualitativa las representaciones y juicios de los estudiantes, en torno a las modalidades y prácticas evaluativas empleadas en la asignatura de Historia, Geografía y Ciencias Sociales. 
Para alcanzar dicho objetivo en general este estudio parte de la premisa de comprender los fenómenos desde su propia lógica interna, lo que fluye de un enfoque cualitativo (Noboa \& Robaina, 2016). Para su logro se han planteado los siguientes objetivos específicos:

a) Explorar las representaciones y juicios en los estudiantes en torno a la planficación de la evaluación.

b) Explorar las representaciones y juicios en los estudiantes en torno a la ejecución de la instancia evaluativa.

c) Explorar las representaciones y juicios en los estudiantes en torno a los resultados de la evaluación.

Como se ha mencionado anteriormente, la investigación es de carácter cualitativa, ya que busca en el establecimiento establecer patrones de sentido, por medio del método interpretativo, el cual sitúa el conocimiento históricamente y toma en cuenta la implicación del investigador en el fenómeno estudiado (Flick, 2007).

En este marco, se optó por una estructura metodológica que facilita la retroalimentación permanente entre ámbito de estudio e investigador, estableciendo una relación dialógica y de mutua implicación (Gerber, 2014), abordando el fenómeno estudiado desde el paradigma de la complejidad. De esta manera se permite que surja inductivamente una base teórica para la comprensión de lo estudiado bajo una perspectiva situada y bajo un método interpretativo (Flick, 2007).

El diseño metodológico adoptado será de tipo hermenétuico, el cual se basa en las premisas del enfoque cualitativo, dado que se busca en el establecimiento establecer patrones de sentido en las narrativas expresadas por los participantes a través de las herramientas del método interpretativo, el cual sitúa el conocimiento históricamente y toma en cuenta la implicación del investigador en la interpretación de los fenómenos discursivos estudiados (Flick, 2007).

La muestra fue elegida atendiendo al criterio de transferibilidad a fin de contar con una descripción exhaustiva y profunda de cada fenómeno en su contexto. Por tanto, la muestra elegida busca lograr un conocimiento profundo y detallado de y sobre los casos en los que tiene lugar el fenómeno de interés, generalizable para otras situaciones en las que dicho fenómeno ocurre.

Las instituciones seleccionadas son nueve (09), uno de enseñanza básica y ocho de enseñanza media, las cuales se encuentran en estado de vulnerabilidad (El promedio IVE-SINAE en Educación Básica y Enseñanza Media de la región es de 79,3\%) y en zona fronteriza, configurando un espacio de intercambio multicultural. En relación a su ubicación, los centros escolares se ubican en distintos paisajes geográficos de la región ( zona costera-valles) y (urbanos y rurales).

Asimismo, se aplicó un muestro no probabilístico en su variante por conveniencia, atendiendo a los objetivos de la investigación y los recursos disponibles por el investigador.

Tabla 1

Entrevista en Profundidad

\begin{tabular}{ccc}
\hline Entrevista a estudiantes & \multicolumn{2}{c}{ Nivel Educacional } \\
\hline 9 & Enseñanza Básica & Enseñanza Media \\
\hline
\end{tabular}

Fuente: elaboración propia (2020)

La presente investigación ha respetado las reglas de confidencialidad y privacidad de los contenidos ofrecidos por parte de las unidades de análisis en las entrevistas desarolladas. Asimismo, en razón de la edad de los participantes y en consideración de los tratados internacionales en materia de 
investigación científica, se han tomado en cuenta los preceptos señalados en la Declaración de Helsinki (1964) en atención a la petición de consentimiento de los responsables de los menores de edad y lo vertido en el Informe Belmont en (1978) en relación al trato hacia los menores de edad en la investigación científica. En ese sentido, el comité científico de la Universidad de Tarapacá (CEC) valida los documentos de "Cartas de consentimientos para participación de hijos" y el " Asentimiento informado".

En relación al procedimiento de diseño y validación del instrumento, un elemento transcendental para el éxito del trabajo de campo, se cimienta en el proceso de diseño y validación de los instrumentos, que se utilizarán en la investigación, entendiendo que la técnica a utilizar es la entrevista semiestructurada. Esta fue construida considerando ochos preguntas abiertas, las cuales han sido estructuradas a partir del concepto "prácticas evaluativas" el cual ha sido delimitado en la literatura de la especialidad. Estas se agruparon en tres categorías propias del ciclo del proceso evaluativo, como lo es la planificación, ejecución y entrega de resultados de la evaluación. En esta etapa, la validación de expertos fue vía correo electrónico en donde se adjuntó una plantilla de análisis y el guión con las preguntas. A su vez, el objetivo general y los específicos, indispensable para que los expertos pudiesen analizar y emitir un juicio, se efectúo por el mismo medio.

Se consideraron 4 doctores especilistas en la temática y de diferentes escenarios educativos (España y Colombia).

\section{RESULTADOS}

Parte importante del proceso de evaluación es la planificación, la cual parte de criterios institucionales y estructurales que circundan a la relación pedagógica. En ese sentido, con respecto al nodo "planificación de la evaluación" y en base a las entrevistas realizadas, se puede observar que los estudiantes emiten diferentes juicios alrededor de las finalidades de la evaluación: En primer lugar, conciben los propósitos de la evaluación como un proceso en que se "evalúan", "miden" o "demuestran" capacidades, poniendo énfasis de forma diferenciada, tanto, en el rol activo del docente-evaluador y en el alumno como agente-activo que "demuestra sus capacidades".

Asimismo, la prueba escrita es el tipo de evaluación más utilizada, lo cual nos insta a pensar en la evaluación como un espacio de verificación del conocimiento adquirido a lo largo del periodo académico. Cabe mencionar, que sólo en ocasiones, se utilizan actividades evaluativas como la disertación y la elaboración de mapas conceptuales.

Por tanto, se asociaría el uso de la evaluación tradicional con motivaciones centradas en la comodidad y conveniencia del profesor y la escasa significatividad desde la óptica del esfuerzo del estudiante. Por otra parte, el uso de otros tipos de evaluación, se asociaría con la intención de realizar una evaluación en profundidad, lo que, desde esta perspectiva el alumno significaría un desarrollo de otras habilidades cognitivas.

Es en la ejecución de la evaluación en que se da la mayor parte de interacciones entre el evaluador y el alumno, de esta manera, de las entrevistas realizadas se pueden perfilar un estilo abierto al momento de dar las instrucciones acerca de la evaluación.

Las sanciones y prohibiciones están relacionadas con conductas que eventualmente podrían sabotear los objetivos de la evaluación.En ese sentido, basándonos en las entrevistas realizadas, es clara la presencia de los aparatos móviles en la dinámica escolar, es su uso lo que podría perjudicar el desenvolvimiento de los procesos evaluativos.

Asimismo, se resalta que las saciones van desde el retiro del examen hasta la llamada a los apoderados. En base a las entrevistas podemos señalar la presencia de una clara distinción entre la representación del docente como autoridad-sancionadora y la de docente-colaborador. Siendo así, 
la primera se asocia con la de un agente vigilante del orden interno dentro del aula, mientras que la segunda se asociaría a la de un agente orientador que clarifica las dudas de los estudiantes ante un punto oscuro de la evaluación, dichas representaciones van asociadas a actitudes y movimiento corporal del evaluador dentro del aula

En relación al dominio de entrega de resultados de la evaluación, la representación que los entrevistados poseen acerca de la nivelación de los aprendizajes tiene dos particularidades: primero, es flexible, ya que se permite a los alumnos poder mejorar las notas obtenidas en el examen; segundo, los mecanismos de nivelación se basan en una evaluación a nivel individual o nivel grupal de las habilidades adquiridas.

Desde la perspectiva del alumnado, el proceso de aprendizaje concluiría con la nota asignada. Así, los que no obtuvieron el puntaje aprobatorio deberán rendir evaluaciones supletorias o trabajos adicionales para el nivelamiento. La percepción del alumnado incide en que el proceso de aprendizaje lejos de concebirse en forma dinámica y bidireccional se traduce en un proceso lineal, acabado y que cuentra su culminación en un puntaje asignado, en base a lo cual, las estrategias de nivelación se llevan a cabo sin cuestionar la naturaleza lineal del procedimiento.

De esta forma, algunas estrategias para remediar los resultados más bajos, se producen a partir de la asignación de trabajos o reforzamientos en clase. Por medio de las entrevistas se ha podido observar que se da mayor preponderancia a la evaluación sumativa, tanto para las evaluaciones principales como para los remediales posteriores a la calificación. Es evidente la relación con la mejora de los resultados a través de trabajos, los cuales se administrarán en función a la cantidad de alumnos con calificaciones insatisfactorias.

\section{DISCUSIÓN}

De los hallazgos obtenidos podemos observar que el aspecto actitudinal se encuentra latente en el aprendizaje como procesos de subjetivación en la evaluación por parte del docente. Estos hallazgos coinciden con lo presentado por Borjas (2016) y Lipman (2016) quienes sostienen que los procesos de aprendizaje-evaluación tienen como eje la subjetividad expandida, la cual implica un acto de apropiación de los contenidos brindados por el docente. Asimismo, se reflexiona acerca del aprendizaje como proceso unidireccional y receptivo, el cual se basa en una imagen científica de mundo, actitud que se trasunta a la relación alumno-docente.

La representación acerca de los procesos de evaluación y los criterios para la nivelación han evidenciado la presencia de una dimensión actitudinal, hallazgos que coinciden con los reportados por Fonseca (2011) quién observó que el momento pedagógico se ve influido por factores extracurriculares y actitudinales provenientes tanto de las condiciones estructurales que atraviesan los agentes como los juicios valorativos de alumnos y docentes. Ello corrobora el juicio que existen variables extracurriculares que influyen en el proceso de aprendizaje y evaluación, por lo que deberá ser tomado en cuenta para una renovación de las estrategias.

Los hallazgos apuntan a un fuerte sentido de realidad otorgado a los exámenes escritos, lo que se condice con lo presentado por Borjas (2016) y Lipman (1997) quienes sostuvieron que, dentro del esquema científico del mundo, la subjetividad del alumno es entendida como ruido, interferencia o error, por tanto, la función de la evaluación es reducir al mínimo las fuentes de perturbación; en este proceso, el docente evaluador se encargará de preservar la validez del proceso evaluativo. En ese ese sentido, la renovación de las estrategias evaluativas pasa por revalorar la percepción que tiene el alumnado con respecto al conocimiento que internaliza. De esta forma, en la evaluación debe alejarse de la sobredimensionalización en el momento de la evaluación y priorizar el momento pedagógico. 
Los hallazgos apuntan a señalar el remedio de los resultados de la evaluación como la corrección normativa de los aprendizajes insuficientemente obtenidos, lo que refuerza el sentido real que los alumnos le atribuyen a la evaluación, preponderantemente escrita. No obstante, esta subsanación crea espacios en que la subjetividad del alumnado se entrecruza con los juicios actitudinales de los docentes dando pie a una concepción dinámica de la evaluación. Esto, da luz acerca de que la renovación de las estrategias de evaluación se han producido ya en los espacios institucionalizados desde la misma acción social, de abajo hacia arriba.

Finalmente, entre las principales limitaciones del presente estudio deben mencionarse que, si bien el planteamiento del problema es delimitado y específico, este es poco flexible y, por tanto, sólo se busca los necesarios en función a los objetivos de la investigación. El diseño de la investigación limita la recolección de datos más generales y otras actividades que puedan incluirse en la investigación.

\section{CONCLUSIONES}

En relación a la información expuesta, se puede evidenciar representaciones consolidadas en los estudiantes con respecto a los procesos evaluativos en establecimientos públicos, vulnerables y fronterizos. Destaca que predomina la evaluación escrita y sumativa como el instrumento más utilizado por los docentes, sin embargo, no es precisamente la de mayor preferencia para los estudiantes.

A su vez, los estudiantes representan a la evaluación como una corroboración de contenidos, dichas percepciones también se enlazan con criterios extra-institucionales, los cuales hacen referencia a la actitud de los docentes y procesos afectivos producto de la interacción profesoralumno. Ello, sin duda reviste de importancia toda vez que el alumnado considera que la evaluación formativa supone más trabajo para el docente.

Con respecto a las representaciones acerca de la ejecución de la instancia educativa, la exploración realizada apunta a que las representaciones y juicios acerca del docente son, por un lado, el de un agente-sancionador, preocupado por el mantenimiento del orden dentro de la sala de clase, asi como de sancionar conductas que puedan sabotear los objetivos del proceso de evaluación de los aprendizajes. Ello, reviste de importancia toda vez que nos da luz acerca del componente motivacional bajo el cual se percibe la ejecución de la evaluación por parte del alumnado.

Con respecto a las representaciones acerca de la resultados de la evaluación, la exploración realizada apunta a que las representaciones y juicios son cambiantes, toda vez que el docente flexibiliza los mecanismos de evaluación, a fin de que se les asigne trabajos acorde a sus habilidades. Ello reviste de importancia, dado que, desde la óptica de los alumnos, el proceso de aprendizaje lejos de concebirse en forma dinámica y bidireccional se traduce en un proceso lineal, acabado y que encuentra su culminación en un puntaje asignado, lo que es solvente en el contenido crítico de la percepción subjetiva de los alumnos en relación a las estrategias de evaluación. En este sentido, la forma y procedimientos que asume la evaluación está fuertemente ligada al perfil del estudiante, por lo que su vulnerabilidad marca el proceso, generando una suerte de expectativas poco ambiciosas en los docentes y condicionando la modalidad e instrumentos empleados.

Por otro lado, en general, los reglamentos de evaluación consideran la incorporción de distintas modalidades evaluativas, sin embargo, a raíz de lo evidenciado, existe una dicotomía entre lo escrito y lo implementado en los procesos evaluativos.

A su vez, se concibe la subsanación de los resultados bajo la óptica de una metodología sumativa, de la misma forma que la evaluación principal. Sin embargo, es una evaluación flexible que toma en cuenta criterios, tanto actitudinales como de grupo. Se puede observar que el remedio de los 
resultados se representa como la corroboración de los aprendizajes obtenidos lo que refuerza el sentido de realidad que los alumnos le atribuyen a la evaluación, preponderantemente escrita.

En relación con lo anterior, la forma que adquiere la evaluación sumativa se traduce en modalidades tradicionales tipo "lápiz y papel”, y muy vinculado al desarrollo de contenidos, y no al desarrollo de habilidades generales y las propias de la asignatura.

Finalmente, se debe profundizar en la importancia que reviste la evaluación en los procesos de aprendizajes. Es en este sentido, que debe existir una preocupación en torno a los elementos de calidad presentes en las tres etapas del proceso evaluativo ( planificación- ejecución y entrega). Lo anterior, es fundamental para cualquier institución educativa, pero en el caso de los establecimientos vulnerables reviste mayor urgencia. Ya que al tener un contexto vulnerable, existen elementos que deben ser subsanados y que requieren de mayor apoyo, es por ello que se deben establecer altas expectativas con los estudiantes y particularmente en los procesos evaluativos, considerando que hoy debemos ser inclusivos y considerar las distintas modalidades evaluativas para todas y todos los estudiantes. Con ello, se contrubuirá a entregar una educación de calidad y una posibilidad concreta de lograr competencias para la vida.

\section{REFERENCIAS BIBLIOGRÁFICAS}

Borjas, M. (2016). La evaluación del aprendizaje como compromiso: una visión desde la pedagogía crítica. Rastros Rostros, 16(30), 35-45.doi:http://dx.doi.org/10.16925/ra.v16i30.816

Flick, U. (2007). El diseño de la investigación cualitativa. Wahington D.C.: SAGE.

Fonseca, A. (2011). Metodologías participativas, subjetividades expandidas y transdisciplinariedad. Tesis Psicológica, (6), 52-72. Recuperado el 04 de 01 de 2020, de https://www.redalyc.org/pdf/1390/139022629004

Gerber, M. (2014). Complejidad: teoría y método. Santiagod de Chile: Universidad de Chile.

Larrosa, J. (2016). Experiencias (y alteridad) en educación. Revista de Ciencias Educativas de la Universidad Los Rios de Uruguay(XVI(3)), 32-45. Recuperado el 02 de Enero de 2020.

Lipman , M. (1997). Pensamiento complejo y educación. Madrid: Ediciones de la Torre.

Marín, L. (2016). Informe diagnóstico preliminar necesidades de desarrollo profesional docente situado región de Arica y Parinacota. Comité local de Arica y Parinacota. Programa de Comités Locales Área de Desarrollo Profesional Docente. CPEIP, Arica-Chile.

Noboa, A., \& Robaina, N. (16 de noviembre de 2016). Complejidad, estrategias de investigación-acciónparticipación y cambio social. Memoria académica UNLP, 11-21. Recuperado el 05 de 04 de 2020, de http://www.memoria.fahce.unlp.edu.ar/trab_eventos/ev.8533/ev.8533 